\title{
The Formation and Essence of Quasars and Their Connections with Galaxies
}

\author{
Cuixiang Zhong \\ Department of Physics, Jiangxi Normal University, Nanchang, China
}

Email address:

cuixiang_zhong@163.com

To cite this article:

Cuixiang Zhong. The Formation and Essence of Quasars and Their Connections with Galaxies. American Journal of Astronomy and Astrophysics. Vol. 6, No. 1, 2018, pp. 26-30. doi: 10.11648/j.ajaa.20180601.14

Received: March 3, 2018; Accepted: March 19, 2018; Published: April 10, 2018

\begin{abstract}
The discovery of quasars is one of the four great discoveries of astronomy in 1960s. Although the discovery of quasars is very interesting, but it has also brought some difficult problems for human being: are quasars stars or galaxies? Why the luminosity of the most powerful quasars is thousands of times greater than the luminosity of a large galaxy such as the Milky Way whose size is larger than the quasars by millions of times? Why the observed quasars are distant objects? If the redshift of a quasar is cosmic redshift? These problems have long plagued the people in suspense. Fortunately, the author of this paper, through studying the formation and orbit-variation of satellites, planets and stars, has put forward a new theory of galactic structure, therefore revealing the hierarchical structure of galaxies and the existence and characteristics of black holes as the main nodes of galactic structure, especially revealing the formation and essence of quasars and solving all the problems mentioned above. The author found that quasar is essentially a supermassive black hole covered by an extreme thick and dense atmosphere. When such a black hole revolves around its progenitor and whirls on its axis fast, lots of cloud clumps would bump each other to cause immense electricity and thunder, forming strong radio source and high luminosity. The mass of the ancestral quasar billions of light years away from the earth is about 1000 times the total mass of the entire Milky Way galaxy, so its energy output would be 1000 times more than the energy released by the entire Milky Way galaxy. Since a quasar revolves around its progenitor and whirls on its axis fast, therefore the redshift of a quasar is indeed cosmic redshift.
\end{abstract}

Keywords: Galaxies Formation, Galaxies: Structure, Black Holes, Quasars, High-Luminosity, High-Redshifts

\section{Introduction}

In the 1950s, the application of radar technology in astronomical observation promoted the development of radio astronomy, and a series of important results have been obtained since then. Among these results, the discovery of quasars is particularly surprising. In 1960, American astronomer Allan Sandage discovered a radio source (3C48) by using optical telescope, its corresponding optical object is a star like object with a very dark nebula around it, and its star spectrum containing many unknown broad emission lines. [1] In 1962, astronomer discovered another radio source (3C273), and later some people found its corresponding optical object is also a star like object with a dark nebula around it. In 1963, American Holland astronomer Schmidt filmed the optical spectrum of the star like objects, he found that this spectrum was similar with the spectrum of $3 \mathrm{C} 48$ and had the same strange emission lines. [2] Schmidt realized that these were actually spectral lines of hydrogen redshifted at the rate of 15.8 percent. This discovery showed that $3 \mathrm{C} 273$ was receding at a rate of $47,000 \mathrm{~km} / \mathrm{s}$. This discovery revolutionized quasar observation and allowed other astronomers to find redshifts from the emission lines from other radio sources. As predicted earlier by Bolton, 3C 48 was found to have a redshift of $37 \%$ of the speed of light. In 1965, Sandage also found some other quasars, which have the same optical properties as quasi-stellar radio source and have blue color, but have no strong radio emission (maybe it is too weak to be detected), so they are called blue star. No matter whether it is "quasi-stellar radio source" or "blue star", they all have common characteristics, that is, their optical images are similar to stars, but the spectrum shows they are all extra-galactic object with 
large redshifts, so they are called quasars.

Until 1989, astronomers have found thousands of quasars in searching sky at radio and visible wavelengths. Later through digital sky survey, astronomers found nearly 300 thousand quasars. [3] The discovery of quasars is one of the four great discoveries of astronomy in 1960s. Although the discovery of quasars is very interesting, but it has also brought some difficult problems for human being: are quasars stars or galaxies? Why the luminosity of the most powerful quasars is thousands of times greater than the luminosity of a large galaxy such as the Milky Way whose size is larger than the quasars by millions of times? Why the observed quasars are distant objects? If the redshift of quasars is cosmic redshift? These problems have long plagued the people in suspense. Fortunately, the author of this paper, through studying the formation and orbit-variation of satellites, planets and stars, has put forward a new theory of galactic structure, therefore revealing the hierarchical structure of galaxies and the existence and characteristics of black holes as the main nodes of galactic structure. This new theory can be used to reveal the formation and essence of quasars, and solve all the problems mentioned above.

\section{The Formation and Evolution of Galactic Structure}

\subsection{The Formation and Evolution of Planetary Systems}

According to the Solar System's formation law described in some papers of the author, a star can produce several planets around it, and each planet can also produce zero or several satellites around it, therefore forming a planetary system, which is a hierarchical structure.

In a hierarchical galaxy, any star other than root-star was generated as a satellite of its parent planet. These satellites unceasingly incorporated the nebula materials near the orbits to become larger and larger, and gradually moved away from its parent planet under the impact of moving objects or the effect of the centrifugal force produced by the accelerating rotation of its parent planet. Especially, when the parent planet becomes a fixed star, its satellites would become planets. During the planets' revolution around their parent star, they unceasingly absorb the nebula materials near the orbits to become larger and larger and may also generate new satellites. So except some small planets (like Mercury and Venus of the Solar System) near the parent star, other larger planets have their own thick atmospheres. During the normal rotation of a planet (such as Earth) around the parent star (such as the Sun), the atmospheric pressure on the trailing hemisphere of the planet is higher than the other hemisphere, effectively increasing its speed, thus making the planet gradually move away from the parent-star along a spiral line. [4] As the planets gradually move away from their parent star, the gravitational attractions of the parent star to these planets also gradually become smaller, consequently these planets' self-rotation will gradually accelerate, making their satellites' revolution faster, eventually causing the satellites to move away from their planet. In addition, with the growth of the mass of Earth, Earth will shrinks from time to time, but conserving the angular momentum of Earth, so a decrease in the rotational inertia results in an increase in the rotation speed of Earth, and consequently also an increase in the revolution speed of the Moon, making the Moon move away from Earth.

\subsection{The Formation and Evolution of Stars}

During the rotation of a planet around its parent star, it unceasingly absorbed dust and gases near the orbits, making its mass increase gradually and its atmosphere become thicker and thicker. When a planet's mass becomes very large, its core temperature and gas density are high enough to initiate and maintain thermonuclear reactions, a new star is born. After a star is born, it enters an evolutionary phase using hydrogen fusion as its major energy source. The outward expansion force of the gas inside the star and the inward contraction force induced by gravity are roughly balanced, making the star neither contract nor expand. So this is a relatively stable phase, the duration is about $90 \%$ of its whole life span, which is called the main sequence stage of a star, it is the prime of the star's life. [5]

Later, as the preponderance of atoms at the core becomes helium, stars like the Sun begin to fuse hydrogen along a spherical shell surrounding the core. This process causes the star to gradually grow in size, passing through the subgiant stage until it reaches the red giant phase. [6] Stars with at least half the mass of the Sun can also begin to generate energy through the fusion of helium at their core, whereas more-massive stars can fuse heavier elements along a series of concentric shells. Once a star like the Sun has exhausted its nuclear fuel, its core collapses into a dense white dwarf and the outer layers are expelled as a planetary nebula. [7] Stars with around ten or more times the mass of the Sun can explode in a supernova [8] as their inert iron cores collapse into an extremely dense neutron star or black hole. [9] [10]

\subsection{The Hierarchical Structure of Galaxy}

According to the Solar System's formation law described in some papers of the author: a star may have its own parent-star or higher ancestral stars, and can produce several planets around it, and each planet can also produce several satellites around it; after a satellite grows into a planet, it can also produce its next generation of satellites. Hence, the basic structure of a galaxy is a hierarchical structure composed of many generations of stars, like a tree, and the entire universe contains many such galaxies, like an endless forest. In a typical galaxy structure, a path from the galaxy center as the root of the tree to one of the moons as the leaves of the tree, may contain several black holes, several neutron stars, and several white dwarfs, $0 \sim 1$ red giant stars, 1 main-sequence stars, 1 planet and 1 satellite. Each branch of a galaxy usually has at most two layers of luminous stars, and these luminous stars are usually at the bottom of the galaxy. 


\section{The Formation and Evolution of Black Holes}

In fact, according to the galaxy formation theory described above, any galaxy is a hierarchical structure composed of many generations of stars and each generation consists of several brother stars. Such galaxy structure is not static, but in constant motion and change. A star of a later generation in a galaxy always revolves around its predecessors, i.e., a satellite moves around its parent planet, a planet moves around its parent star, and a star may further moves around its parent star, until all the daughter galaxies rotate around the center of the galaxy. During a star revolving around its parent star, it unceasingly incorporates the nebula materials near the orbits to become larger and larger. In addition, for a planet, it may gradually move away from its parent star under the impact of moving objects or the radiation of its parent star, even producing progeny galaxies. This means that a star of the $n$th $(n \geq 1)$ generation might produce a sub-galaxy consisting of multiple generations of stars, making its spatial scale become overlong, so that when the sub-galaxy rotates around the star of the $n$th generation, its tail satellite may enter the gravitational field of the star of the $(n-1)$ th generation, and finally be annexed by the later one, greatly increasing the mass and density of the later one, as is shown in Figure 1.

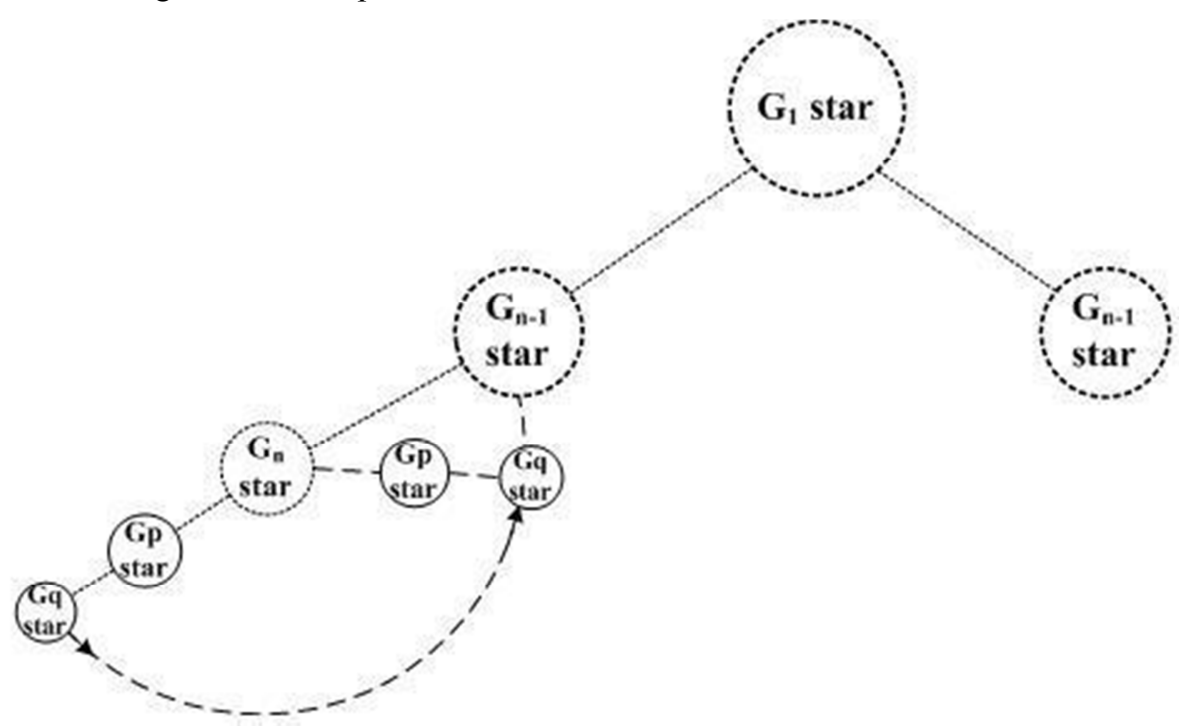

Figure 1. An overlong sub-galaxy impacting on its parent star makes the mass of the parent star and the distance between the two generation stars increase.

Moreover, the impact of the sub-galaxy of the $n$th generation star on the $(n-1)$ th generation star will produce tremendous energy, even start thermonuclear reactions, to fuse the satellite with the $(n-1)$ th generation star. Due to the strong gravitation of the center of the $(n-1)$ th generation star, the newly added material shrinks immediately, but conserving the angular momentum of the $(n-1)$ th generation star, so a decrease in the rotational inertia results in an increase in the rotation speed of the $(n-1)$ th generation star, and consequently also an increase in the revolution speed of the $n$th generation star, making the $n$th generation star move away from the $(n-1)$ th generation star, widening the distance between the two generation stars. Similar impacts may occur multiple times, finally allowing the passage of the sub-galaxy of the $n$th generation star. This is the basic principle of the extension of galactic structure.

Thus, we can see that in some old galaxies which have evolved over a long period of time, there are many high layer stars that have accumulated for a long time and encountered numerous impacts, eventually becoming black holes of great mass and density. Especially, the progenitor at the center of the galaxy has been the earliest and most frequently hit by other celestial objects, and has become a black hole with the largest mass and density. Some daughter stars under or beside the center of the galaxy may also have accumulated for a long time and encountered numerous impacts, eventually becoming massive black holes, but their mass and density are lower than that of their ancestor. [11] Just because the mass of the black hole is very large and the gravitational force is very strong, any moving body entering the gravitational field of the black hole but having lower speed than light speed cannot escape the trap of the black hole. Also because the mass of the black hole is very large and the gravitational force is very strong, it can attract an extreme thick and dense atmosphere, so that the light emitted by objects smaller than black holes cannot penetrate so thick and dense an atmosphere, just as sunlight cannot penetrate thick clouds. Even if a luminous celestial body enters this area, since lots of its gaseous, liquid and solid materials may be swallowed by the black hole, making the object's resources are insufficient to maintain its luminous effect, causing the phenomenon of "light can not escape a black hole".

\section{The Formation and Essence of Quasars}

According to the above theory of the formation of black holes, the mass of the black hole is gradually increased. When a black hole of small mass evolves into a super-massive black hole, its gravitational force acting on other objects will become extremely strong, therefore like the earth, it can 
attract atmospheric particles (such as molecules, atoms and dust particles) to form its own atmosphere. Because the mass of a supermassive black holes is much greater than that of the earth, the thickness and density of cloud around the supermassive black hole is much greater than the thickness and density of thunder storm around the earth, so the observers have found that star like objects have very dark nebula around them. When such a black hole revolves around its progenitor and whirls on its axis fast, lots of cloud clumps would bump each other to cause immense electricity and thunder, forming strong radio source and blast wave, so people on the earth can detect remote radio source and cosmic background radiation. In addition, these immense electricity also cause interlaced currents around the dense clouds, which then produce aurora like intense light around the quasar, so quasar is among the most luminous, powerful, and energetic objects known in the universe. That is a quasar, which is essentially a supermassive black hole that is covered by an extreme thick and dense atmosphere and revolves around its progenitor and whirls on its axis fast. Since a supermassive black hole is usually a central star of a large galaxy, it normally has at least two spiral arms. So, when it revolves around its progenitor and whirls on its axis fast, it remotely looks like an active galactic nucleus. [12]

Although a quasar consists of a supermassive black hole surrounded by an orbiting nebula, no quasar has been found in the range of tens of millions of light-years in the neighborhood of the Milky Way. This means that the mass of the largest black hole in the center of the galaxy is not enough to attract thick and dense clouds to generate a wide range of continuous violent thunderstorms. Hence, the mass of a black hole forming a quasar must be far greater than the mass of the largest black hole in the center of the Milky Way, so such a black hole should be at a higher layer in the super-cluster of galaxies than the black hole in the center of the Milky Way. Hence, it is far away from the Earth located at the bottom of the Milky way. In fact, they are usually billions of light years or tens of billions of light years away from Earth.

According to the formula calculating the distance between

$$
5 \times 10^{4}+5 \times 10^{4}+2 \times 5 \times 10^{4}+2^{2} \times 5 \times 10^{4}+\cdots+2^{n-2} \times 5 \times 10^{4}=10^{10} \rightarrow 2^{n-1}=2 \times 10^{5}
$$

thus

$$
M_{n}=2^{n-1} M_{1}=2 \times 10^{5} \times 0.005 m=1000 m
$$

i.e., the mass of the ancestral quasar billions of light years away from the earth is about 1000 times the total mass of the entire Milky Way galaxy, so it is massive enough to attract extreme thick and dense cloud, when it revolves around its progenitor and whirls on its axis fast, a wide range of continuous violent thunderstorms can be produced, the energy released by the quasar would be 1000 times more than the energy released by the entire Milky Way galaxy.

\section{Quasars' Connections with Galaxies}

Since most quasars are at very high redshift, $[14,15]$ the central black hole of a galaxy and the central black hole of one of its sub-galaxy, and the ratio of the central black-hole mass to galaxy mass, we can easily calculate the ratio of the mass of a quasar billions of light years away from Earth to the total mass of the Milky Way. Since a normal galaxy is a hierarchical structure and astronomers have discovered that our galaxy is a suburb of a supercluster of 100,000 large galaxies they have called Laniakea, we can assume that the Milky Way's central black hole has multiple ancestral black holes. Assume there is $n$ black holes from the Milky way's central black hole to a quasar billions of light years away from Earth, numbered respectively as $\mathrm{BH}_{1}, \mathrm{BH}_{2}, \cdots, \mathrm{BH}_{\mathrm{n}}$, and their correspondingly masses are $M_{1}, M_{2}, \ldots, M_{n}$. Let the total mass of the Milky Way be $m$, then the mass of the Milky way's central black hole is $M_{1}=0.005 m$. [13] Just as the Milky Way has two spiral arms, a large galaxy normally has two spiral arms, so the total mass of the galaxy centers on $\mathrm{BH}_{2}$ should be $2 \mathrm{~m}$, the mass of $\mathrm{BH}_{2}$ should be $M_{2}=0.005 \times(2 m)=2 M_{1}$. Similarly, we have

$$
M_{3}=2 M_{2}=2^{2} M_{1}, \quad M_{4}=2^{3} M_{1}, \cdots, M_{n}=2^{n-1} M_{1}
$$

Because the diameter of the Milky Way is $10^{5}$ light-years, its radius is $5 \times 10^{4}$ light-years. In order to make the Milky Way revolving around its central black-hole $\mathrm{BH}_{1}$ not collide with $\mathrm{BH}_{2}$, the distance between $\mathrm{BH}_{1}$ and $\mathrm{BH}_{2}$ must be greater than $5 \times 10^{4}$ light-years, and this distance does not change for a certain period of time. So the distance between $\mathrm{BH}_{1}$ and $\mathrm{BH}_{2}$ is about $5 \times 10^{4}$ light-years. Similarly, the distance between $\mathrm{BH}_{2}$ and $\mathrm{BH}_{3}$ is about $2 \times 5 \times 10^{4}$ light-years, the distance between $\mathrm{BH}_{3}$ and $\mathrm{BH}_{4}$ is about $2^{2} \times 5 \times 10^{4}$ light-years, the distance between $\mathrm{BH}_{n-1}$ and $\mathrm{BH}_{n}$ is about $2^{n-2} \times 5 \times 10^{4}$ light-years. Since the earth is at the outskirt of the Milky Way, and it is billions of light years away from the quasar, hence

that implies they revolve quickly around their parent stars. So, a quasar at high redshift usually has a series of progenitors, and each of its progenitors has two or more spiral arms. In addition, a quasar is essentially a supermassive black hole at the center of a galaxy of large-scale structure, it normally has at least two spiral arms. Since the quasar whirls on its axis fast, its spiral arms or sub-galaxies also follow the quasar's rotation to move quickly around it. In order to allow the quasar moving around its parent star and its sub-galaxies moving around itself, the distance between the the quasar and its parent-star should be larger than half the diameter of the host galaxy of the quasar. Hence, if the distance between the earth and a quasar is $10^{10}$ light-years, which is about the radius of the host galaxy of the quasar, then the distance between the 
quasar and its parent star should be larger than $10^{10}$ light-years, therefore the distance between the earth and the quasar's parent star should be larger than $2 \times 10^{10}$ light-years.

\section{Conclusions}

Microwave background radiation, pulsar, quasar and interstellar molecules were regarded as four great discoveries of astronomy in the 1960s, especially the discovery of quasars is very interesting, but it has also raised some questions for people. Fortunately, the author of this paper, through studying the formation and orbit-variation of satellites, planets and stars, has put forward a new theory of galactic structure, therefore revealing the hierarchical structure of galaxies and the existence and characteristics of black holes as the main nodes of galactic structure. This new theory can be used to reveal the formation and essence of quasars, and solve all the problems mentioned above.

\section{References}

[1] Matthews T A, Sandage, A R. ApJ, 1963, 138:30.

[2] Schmidt M. Nature, 197:1040.

[3] Wu X B, Wang F, Fan X et al. Nature, 2015, 518:512.
[4] Cui-xiang Zhong, The Real Reason for the Earth and Other Planets to Move Away from the Sun as Well as Natural Measures to Mitigate Global Warming, 2016, International Journal of Geophysics and Geochemistry.

[5] Sackmann, I.-J., et al., 1993, Our Sun. III. Present and Future, ApJ, 418, 457.

[6] Schawinski, K., et al., 2008, Supernova Shock Breakout from a Red Supergiant, Science, 223, 226.

[7] Laughlin, G., et al., 1997, The End of the Main Sequence, ApJ, $420,426$.

[8] Krause, O., et al., 2008, The Cassiopeia A Supernova was of Type IIb, Science, 1195, 1197.

[9] Brazier, K. T. S., et al., 2013, The implications of radio-quiet neutron stars, MNRAS305, 671.

[10] O'Leary, R. M., et al., 2006, Binary Mergers and Growth of Black Holes in Dense Star Clusters". ApJ, 637, 937.

[11] Kermendy, J. \& Ho, L. C. Annu. Rev. Astron. Astrophys. 2013, 51, 511:653.

[12] Häiring, N. \& Rix, H.-W. ApJ, 2004, 604, L89-L92.

[13] Venemans, B. Nature, 2015, 518:512.

[14] Alam S, Albareti F D, Prieto C A et al. ApJS, 2015, 219:12.

[15] Wang F G, Wu X B, Fan X et al, ApJL, 2015, 807:L9. 